\title{
Real-time fMRI for brain-computer interfacing
}

Citation for published version (APA):

Sorger, B., \& Goebel, R. (2020). Real-time fMRI for brain-computer interfacing. Handbook of Clinical Neurology, 168, 289-302. https://doi.org/10.1016/B978-0-444-63934-9.00021-4

Document status and date:

Published: 01/01/2020

DOI:

10.1016/B978-0-444-63934-9.00021-4

Document Version:

Publisher's PDF, also known as Version of record

Document license:

Taverne

\section{Please check the document version of this publication:}

- A submitted manuscript is the version of the article upon submission and before peer-review. There can be important differences between the submitted version and the official published version of record.

People interested in the research are advised to contact the author for the final version of the publication, or visit the DOI to the publisher's website.

- The final author version and the galley proof are versions of the publication after peer review.

- The final published version features the final layout of the paper including the volume, issue and page numbers.

Link to publication

\footnotetext{
General rights rights.

- You may freely distribute the URL identifying the publication in the public portal. please follow below link for the End User Agreement:

www.umlib.nl/taverne-license

Take down policy

If you believe that this document breaches copyright please contact us at:

repository@maastrichtuniversity.nl

providing details and we will investigate your claim.
}

Copyright and moral rights for the publications made accessible in the public portal are retained by the authors and/or other copyright owners and it is a condition of accessing publications that users recognise and abide by the legal requirements associated with these

- Users may download and print one copy of any publication from the public portal for the purpose of private study or research.

- You may not further distribute the material or use it for any profit-making activity or commercial gain

If the publication is distributed under the terms of Article $25 \mathrm{fa}$ of the Dutch Copyright Act, indicated by the "Taverne" license above, 


\title{
Real-time fMRI for brain-computer interfacing
}

\author{
BETTINA SORGER ${ }^{1,2}$ AND RAINER GOEBEL ${ }^{1,2 *}$ \\ ${ }^{I}$ Department of Cognitive Neuroscience, Maastricht University, Maastricht, The Netherlands \\ ${ }^{2}$ Maastricht Brain Imaging Center (M-BIC), Maastricht, The Netherlands
}

\begin{abstract}
Brain-computer interfaces (BCIs) based on functional magnetic resonance imaging (fMRI) provide an important complement to other noninvasive BCIs. While fMRI has several disadvantages (being nonportable, methodologically challenging, costly, and noisy), it is the only method providing high spatial resolution whole-brain coverage of brain activation. These properties allow relating mental activities to specific brain regions and networks providing a transparent scheme for BCI users to encode information and for real-time fMRI BCI systems to decode the intents of the user. Various mental activities have been used successfully in fMRI BCIs so far that can be classified into the four categories: (a) higher-order cognitive tasks (e.g., mental calculation), (b) covert language-related tasks (e.g., mental speech and mental singing), (c) imagery tasks (motor, visual, auditory, tactile, and emotion imagery), and (d) selective attention tasks (visual, auditory, and tactile attention). While the ultimate spatial and temporal resolution of fMRI BCIs is limited by the physiologic properties of the hemodynamic response, technical and analytical advances will likely lead to substantially improved fMRI BCIs in the future using, for example, decoding of imagined letter shapes at $7 \mathrm{~T}$ as the basis for more "natural" communication BCIs.
\end{abstract}

\section{USING fMRI TO MEASURE BRAIN ACTIVATION}

Since its invention almost 30 years ago (Ogawa et al., 1990), functional magnetic resonance imaging (fMRI) has become one of the most widely used and, probably, publicly the most visible noninvasive technique to measure brain activation. fMRI has played a central role in the development of cognitive neuroscience, and several new fields, including social neuroscience, neuroeconomics, and genetic imaging. The strengths of this technique are its spatial resolution, the ability to reach deep subcortical structures, and whole-brain coverage, enabling the mapping of functionally connected networks and the extraction of information from activation patterns that are distributed across different brain regions. In the psychiatric domain, fMRI has made major contributions to the understanding of psychopathology, and in neurology, fMRI it has become a central technique for mapping neuroplasticity, e.g., in recovery from stroke (Seitz, 2010) and for presurgical mapping in the context of tumor and epilepsy surgery.

\section{Physiologic principles of fMRI}

fMRI is currently the predominant method to study brain activation as it provides an (indirect) measure of neuronal activity with high spatial and good temporal resolution (Logothetis et al., 2001) following external or internal stimulation (e.g., mental task performance). Task-related increased neuronal activity causes a localized change in cerebral metabolic rate of oxygen $\left(\mathrm{CMRO}_{2}\right)$, cerebral blood flow (CBF), and cerebral blood volume (CBV). Consequently, the concentration ratio of oxygenated and deoxygenated hemoglobin changes. Because of the different magnetic properties of oxy- and deoxygenated

\footnotetext{
*Correspondence to: Rainer Goebel, Department of Cognitive Neuroscience, Faculty of Psychology and Neuroscience, Maastricht University, Postbus 616, 6200 MD, Maastricht, The Netherlands. Tel: +31-0-43-388 4014, Fax: +31-0-43-3884125, E-mail: r.goebel@maastrichtuniversity.nl
} 
hemoglobin (dia- and paramagnetic, respectively), the change in the concentration ratio gives rise to the measurable blood oxygenation level-dependent (BOLD) effect (Ogawa et al., 1990). The BOLD contrast, thus, reflects the combined effect of several physiologic changes, which are linked to neuronal activity through neurovascular coupling. After a short (e.g., 100-ms) event, the typically observed hemodynamic response function (HRF) is characterized by an initial dip, a rapid increase of the signal peaking around $4-8 \mathrm{~s}$ followed by a slower decrease, and, in most cases, an undershoot before returning to baseline. Fig. 21.1 shows a typical fMRI response to a longer event (10-s finger tapping). Due to the nature of the fMRI signal, the ultimate limit of both temporal and spatial resolution is imposed by the temporal (in the range of seconds) and spatial (in the range of hundreds of microns) resolution of the hemodynamic response. With standard 1.5- and 3-T scanners, fMRI signals are typically measured with a resolution of $1-3 \mathrm{~mm}$ in all three spatial dimensions and a temporal resolution of 1-3 s. With ultrahigh field fMRI scanners (7 T and higher) submillimeter spatial resolution is achievable allowing to separate signals from cortical layers and cortical columns (De Martino et al., 2015). While recent developments in MR physics such as simultaneous multislice MRI pulse sequences (also known as "multiband" sequences) allow fast acquisition of whole-brain fMRI datasets (Moeller et al., 2010), the hemodynamic delay in the robust BOLD-signal increase limits the use of fMRI BCIs aiming at fast response times. While the "elusive" initial dip (Uludag, 2010) would allow faster detection of neuronal changes, it is unfortunately not robustly detectable, even after substantial (offline) averaging.

\section{Measurement artifacts}

Task-related fMRI measurements show increases of only about $1 \%-5 \%$ with respect to baseline signal levels and are mixed with physical and physiologic noise fluctuations that are roughly similar in size. Furthermore, most fMRI studies are performed using the BOLD sensitive GE-EPI MR pulse sequence because of its speed, but it has the disadvantage that images suffer from signal dropouts and geometric distortions, especially in brain regions close to air and liquor (so-called susceptibility artifacts). These artifacts can be reduced substantially by using optimized EPI sequence parameters (Weiskopf et al., 2006) and parallel imaging techniques. The quality of fMRI data is especially hampered in the presence of substantial head movements. In case of substantial head motion, datasets may become even unusable, either completely or in part. If head movements are small (in the range of a few millimeters of translation/degrees of rotation), three-dimensional (3D) motion correction is an important step to improve data quality for subsequent data analysis. To further enhance signal quality, spatial smoothing may be optionally performed using, e.g., a Gaussian filter with a full-widthat-half-maximum (FWHM) of a few millimeters. Most offline preprocessing routines, including $3 \mathrm{D}$ motion correction, removal of signal drifts and spatial smoothing,

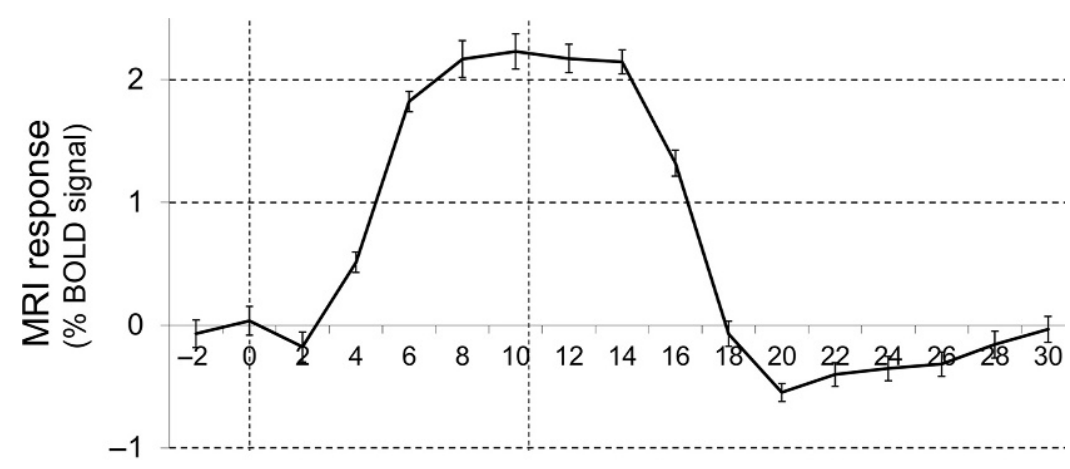

Time (s)

Fig. 21.1. Time course of the hemodynamic response as measured with fMRI. The figure demonstrates the time course of the BOLD-signal change in the left-hemispheric primary motor cortex (M1) of an individual participant performing sequential right-hand finger tapping for $10 \mathrm{~s}$ (interval between vertical dashed lines) with 20-s resting periods in between. At first (after $2 \mathrm{~s}$ ), an "initial dip" is observable resulting from the short transient decrease of the oxygenation level in the small vessels immediately following the onset of neuronal activity. Then, the signal intensity strongly increases ("overcompensation," positive BOLD response), reaching its peak about $10 \mathrm{~s}$ following task onset. After $14 \mathrm{~s}$ ( $4 \mathrm{~s}$ after cessation of task performance), the signal begins to rapidly decay eventually falling below the initial baseline level ("undershoot"). Finally, the fMRI signal slowly returns to baseline reaching it $30 \mathrm{~s}$ after the original task onset. Remarks: data shown are event-related averages across 10 trials performed at a magnetic field strength of $3 \mathrm{~T}$; error bars indicate variance ( \pm s.e.m.). 
are also employed in real-time analyses to increase the fMRI signal-to-noise ratio (SNR). For BCI applications, these preprocessing routines need to be implemented efficiently so that they can be employed as part of a real-time data analysis pipeline. Removal of linear and nonlinear trends is typically not performed as a preprocessing step during real-time processing but are incorporated in the statistical (whole-brain and/or regional) analysis using low-frequency drift predictors in the design matrix of an incrementally estimated general linear model (GLM).

\section{SUITABILITY OF AMRI AS FUNCTIONAL- NEUROIMAGING METHODS IN BRAIN-COMPUTER INTERFACING}

\section{Desired aspects of functional-neuroimaging methods in the context of BCIs}

Naturally, any BCI constitutes a complex and technically challenging system. Still, a general goal of BCI design and development is to create BCI systems that are highly robust, safe, user-friendly, and cost-effective. Fulfilling these requirements will increase the chance that a particular BCI system will be in the end applied to daily-life situations (if necessary) and will be accessible to a wide range of users. In the following section, we evaluate the mentioned requirements with respect to fMRI.

\section{Evaluation of fMRI as BCI Method}

\section{Robustness}

A BCI system is robust if high single-trial decoding accuracies can be reached. In principle, this can be achieved with fMRI due to the relatively high single-trial reliability, high signal-to-noise ratio, and high spatial resolution of the BOLD signal (Sorger et al., 2009, 2012). Moreover, up to $100 \%$ of the brain can be simultaneously measured with fMRI (large brain coverage). Still, several (common) artifacts might hamper signal quality (e.g., head motion and susceptibility artifacts) reducing decoding accuracy. Another drawback of fMRI is its relatively low temporal resolution considerably limiting the information-transfer rate, which reaches currently $\sim 5$ bits/min (Sorger et al., 2012), while with EEGbased communication BCIs information transfer rates (ITRs) up to $\sim 80 \mathrm{bits} / \mathrm{min}$ can be achieved when exploiting stimulus evoked responses (Birbaumer et al., 2008).

\section{FleXibility}

MRI scanners lack portability and are normally located in research institutions and clinical environments. Thus, application in daily-life situations is impossible. However, we think that fMRI-based BCIs have considerable flexibility when it comes to individualization. The numerous possibilities to intentionally modulate hemodynamic responses (see Section "Information encoding in fMRIbased BCIs") come along with a high potential for individualization when designing fMRI-based BCIs.

\section{SAFETY}

fMRI is noninvasive and the technique itself is generally considered safe. However, ferromagnetic objects, which erroneously get into the scanner room, constitute a potential cause of danger. For the same reasons, people are not allowed to have paramagnetic parts in or on their body. Therefore, fMRI participants and researchers operating MRI scanners have to be carefully screened for metal before entering the scanner room.

\section{USER FRIENDLINESS}

User friendliness is a very important BCI requirement. Mostly, two kinds of people are involved in the BCI process. First, the actual BCI user, i.e., the person who is part of the BCI system and wants to employ the BCI system to motor/independently interact with the environment or to monitor/intentionally change their own brain activation. Another person potentially involved is the $\mathrm{BCI}$ operator, mostly an expert (neuroscientist, computer scientist, technical engineer etc.) who supports the BCI user in employing the system. In an ideal situation, a $\mathrm{BCI}$ operator would not be necessary. However, this is so far only possible for extremely basic BCIs. Obviously, an fMRI-based BCI requires at least one very skilled BCI operator who has to simultaneously control at least three personal computers (PCs) (scanner console, stimulation $\mathrm{PC}$, and real-time data analysis PC, see Fig. 21.2). On the side of the $\mathrm{BCI}$ user, there are several facts that limit user friendliness of fMRI-based BCIs. The BCI user is in an extremely unnatural situation: Due to the specific methodology, the user is located in a separate scanner room isolated from other people. This seriously hampers interaction between BCI users and operators. Moreover, the BCI user must remain in a lying position in a tight bore and movements must be avoided to assure sufficient data quality, which makes the method mostly unsuitable for claustrophobics. Also, the unavoidable scanner noise constitutes a considerable additional burden. Finally, some people, especially when exposed to ultra-high magnetic fields, experience unpleasant side effects (e.g., vertigo, phosphenes) (Rauschenberg et al., 2014).

To summarize, the user friendliness of fMRI-based BCIs is rather limited. Maybe one advantage of the fMRI-BCI method in this context is its relatively short preparation time compared to other functional neuroimaging methods because there is no necessity for time-consuming placement of electrodes (electroencephalography; EEG) or optodes (functional near-infrared spectroscopy; fNIRS). 


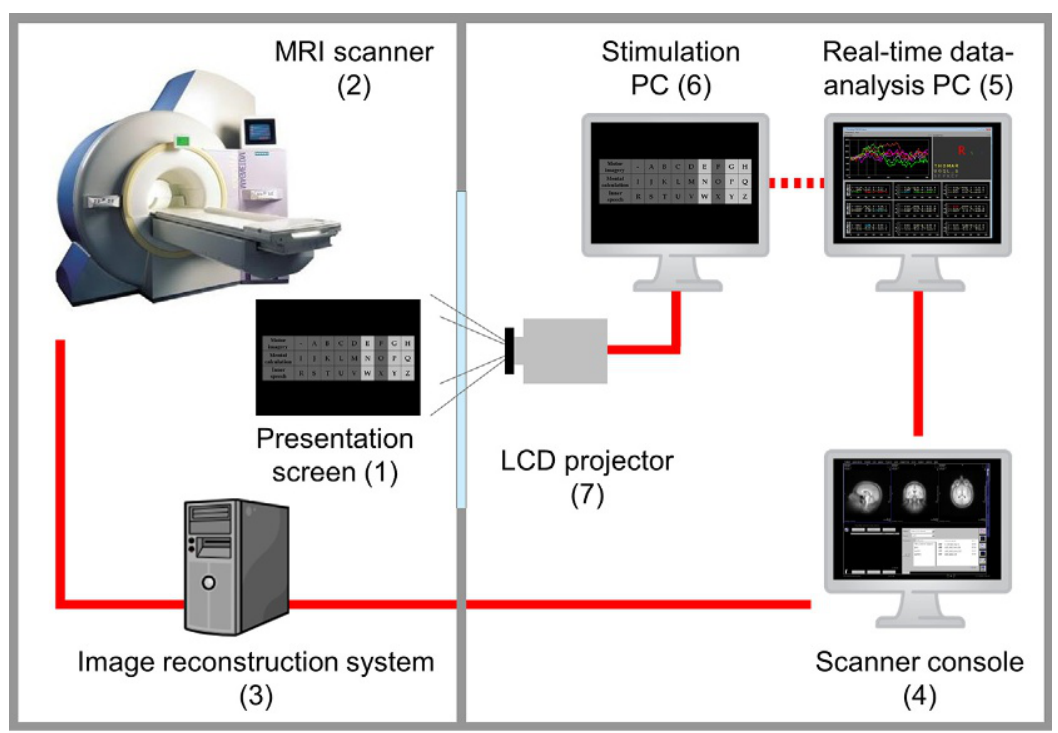

MRI scanner room

MRI scanner console room

Fig. 21.2. Technical setup and data flow in a real time-fMRI letter-spelling experiment. This figure illustrates the components of the technical setup and the different stages of the data flow during an fMRI-based letter-spelling experiment. A participant is visually guided via a presentation screen (1) to encode letters while functional images are being acquired (2). Immediately after, the images are reconstructed in real time (3) and sent to the scanner console's hard disk (4). The PC performing real-time data analysis (5) has instantaneous access to the reconstructed images and the letter-decoding software immediately decodes the encoded letter. In case the participant is provided with information about the decoded letter (not shown), the real-time data-analysis PC (5) is connected to the stimulation PC (6) (dashed red line) and transfers its output (information about the decoded letter) to the stimulation PC. A custom-developed presentation program generates the visual stimulus containing the information about the decoded letter that is presented via the projector (7) on the presentation screen (1). Abbreviations: $L C D$, liquid crystal display; MRI, magnetic resonance imaging; $P C$, personal computer.

\section{Costs}

FMRI is costly when compared to other noninvasive BCIs (EEG, fNIRS). Measurements are associated with high acquisition and maintenance costs as well as with considerable personnel expenses, the latter due to the high level of expertise needed to control an MRI scanner. While it is true that MRI scanners constitute standard medical equipment and are thus used frequently, the use of fMRI as BCI technique will be limited for the aforementioned reasons.

\section{METHODOLOGY OF AMIRI-BASED BRAIN-COMPUTER INTERFACING}

\section{General aspects of information encoding and decoding in brain-computer interfacing}

In a BCI setup, the BCI user (e.g., a "locked-in" patient) communicates a particular intention or message, for example, the answer "yes" or "no," not via an overt behavior (speech, gesture etc.) but through a brain signal. The brain signal is, so to speak, the "carrier" of the information from the inner to the outside world. How can this be achieved?
It would be most convenient, of course, if we could simply use the neural activities (and the resulting brain signals) that are associated with just thinking "yes" or "no." Theoretically, this could be possible as the brain activity that is evoked by thinking "yes" must be at some level different compared to the brain activity caused by thinking "no." However, such activity differences are in all likelihood small and the functional-neuroimaging methods that are available at present are not suited to measure these small differences. As this "direct" information-encoding approach is not feasible, an alternative one has to be employed. Research has shown that people can intentionally and effectively generate differentiable brain activity/brain signals by performing different mental activities. This is exploited in the "indirect" information-encoding approach in which the naturally occurring brain activity is not used but "deputy" brain activity is used. This approach requires implementing a specific translation code shared by the BCI user for encoding and the BCI system for decoding. For example, to encode the answers to a binary (e.g., yes/no) question, the BCI user could, e.g., mentally recite a poem (for encoding "yes") or to imagine to spatially navigate through a house (for encoding "no"). 

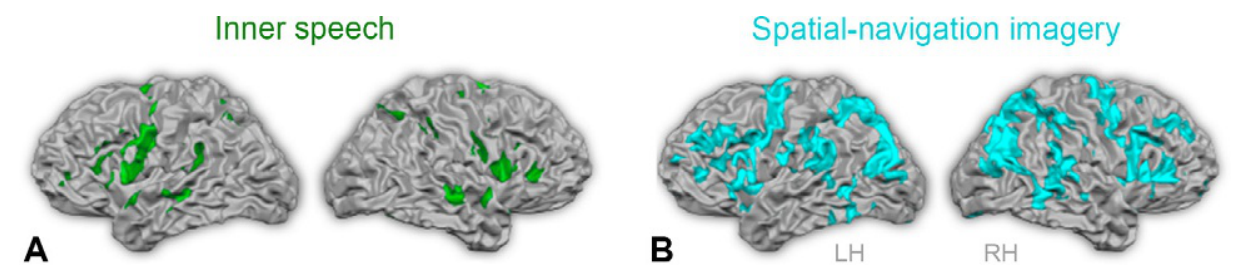

Fig. 21.3. FMRI-activation patterns evoked by two different mental activities. The figure demonstrates the different fMRIactivation patterns evoked by performing (A) inner speech (e.g., mentally reciting a poem) and (B) spatial-navigation imagery (e.g., imagining the three-dimensional scenes while mentally walking through a house). While the activation patterns exhibit some degree of spatial overlap (e.g., within left premotor cortex), most regions are specifically involved in only one of the two mental activities. Abbreviations: $L H$, left hemisphere; $R H$, right hemisphere.

These two mental activities evoke clearly different brainactivity patterns (easily observable when looking at resulting fMRI-activation maps; see Fig. 21.3). The two brain-activity patterns can be obtained in form of the resulting (electric or vascular) brain signals that are measured by either a neuroelectric or a hemodynamic functional-neuroimaging method.

But which indirect information-encoding approach is best-suited for functional-neuroimaging BCIs? There is no clear answer to this question as there are many aspects that have to be considered to answer it. What is important to keep in mind in the current context is that the specific mental activity performed by the BCI user must be reflected to some degree in the brain signal and the functional-neuroimaging method must be sensitive enough to detect this specific brain-signal aspect. This consequently means that for each functional-neuroimaging method specific encoding strategies have to be developed to make the most of the advantages that each method entails.

In Section "Information encoding in fMRI-based BCIs," we introduce mental activities and additional information-encoding strategies which are promising in the hemodynamic BCI context or have even already proven to be successful. Thereafter, in Section "Information decoding using real-time fMRI data analysis," we give an overview of currently available real-time fMRI data analysis (decoding) methods for brain-computer interfacing.

\section{Information encoding in fMRI-based BCIs}

As mentioned before, one important aspect when developing any $\mathrm{BCI}$ is to enable the BCI user to effectively and conveniently translate different intentions and messages (i.e., the to-be-transmitted information) into different mental states resulting in differentiable brain signals. So far, only indirect encoding procedures have been used in real-time fMRI-BCI setups in which the BCI user modulates (a combination of) spatial, temporal, or magnitudinal fMRI-signal features to encode an intention.

\section{Modulation OF SPATIAL fMRI-Signal FEATURES}

One approach to encode separate BCI commands is exploiting the spatial localization of brain functions. By intentionally performing different mental activities, different sets of brain regions get involved resulting in spatially distinct brain-activation patterns. FMRI is especially well suited to obtain these spatially different neural activation patterns as wide parts (up to $100 \%$ ) of the brain can be measured simultaneously. Moreover, fMRI signals demonstrate a high spatial specificity (i.e., the location of the signal source can be well established). This approach has been tested in several fMRI experiments with healthy participants and nonresponsive patients. In a pioneering study (Yoo et al., 2004), healthy participants were asked to navigate through a two-dimensional (2D) virtual maze by performing a specific mental activity (eliciting a unique brain-activation pattern) for each of four movement directions ("right," "left," "up," and "down"). In a later follow-up study, it was demonstrated that this procedure also enables sufficient control over 2D movements of a robotic arm (Lee et al., 2009b). It is important to note that the amount of mental activities that evoke fMRI brain-activation patterns being sufficiently different to be decoded with a high single-trial decoding accuracy (desired in the context of BCI communication and control) is limited to maybe a dozen patterns, at least when using MRI scanners with conventional (clinically available) field strengths (1.5 or $3 \mathrm{~T})$. FMRI at ultra-high magnetic field strengths ( 7 or $9.4 \mathrm{~T}$ ) allows for functional neuroimaging with considerably higher spatial resolution - thereby increasing the chance to differentiate between more similar (but still different) brain-activation patterns. For example, several 7-T fMRI studies have focused on subcategorical decoding of overt behavior (vs mental activities) such as different mouth movements (Bleichner et al., 2015) and hand gestures (Bleichner et al., 2016). So far, maximally six different mental activities were investigated in an individual study (Lee et al., 2009a). Generally, the mental activities that have been tested in the past can be classified into the following four categories: (a) higher-order 
cognitive tasks (e.g., mental calculation (Yoo et al., 2004)), (b) covert language-related tasks (e.g., mental speech and mental singing), (c) imagery tasks (motor, visual, auditory, tactile, and emotion imagery), and (d) selective attention tasks (visual, auditory, and tactile attention). Table 21.1 gives an overview of mental activities investigated in the BCI context applying online or offline decoding.

Table 21.1

Overview of studies exploring and applying various mental activities for fMRI-based brain-computer interfacing

\begin{tabular}{|c|c|}
\hline Reference & $\begin{array}{l}\text { Mental activities investigated for } \\
\text { fMRI-BCI use }\end{array}$ \\
\hline Yoo et al. (2004) & $\begin{array}{l}\text { Mental calculation } \\
\text { Mental speech } \\
\text { Right-hand motor imagery } \\
\text { Left-hand motor imagery }\end{array}$ \\
\hline Sorger et al. (2009) & $\begin{array}{l}\text { Motor imagery (mental drawing) } \\
\text { Mental calculation }\end{array}$ \\
\hline Sorger et al. (2012) & $\begin{array}{l}\text { Motor imagery (mental drawing) } \\
\text { Mental calculation } \\
\text { Mental speech } \\
\text { Mental singing }\end{array}$ \\
\hline Boly et al. (2007) & $\begin{array}{l}\text { Visual imagery (spatial navigation) } \\
\text { Visual imagery (face imagery) mental } \\
\text { singing } \\
\text { Motor imagery (tennis imagery) }\end{array}$ \\
\hline $\begin{array}{l}\text { Owen et al. (2006, } \\
\text { 2007) }\end{array}$ & Visual imagery (spatial navigation) \\
\hline Monti et al. (2010) & Motor imagery (tennis imagery) \\
\hline Yoo et al. (2001) & Auditory imagery \\
\hline $\begin{array}{l}\text { Yoo et al. (2003) } \\
\text { Kaas et al. (2014) }\end{array}$ & Tactile imagery \\
\hline Lee et al. (2006) & $\begin{array}{l}\text { Right-hand motor imagery } \\
\text { Left-hand motor imagery } \\
\text { Right-foot motor imagery } \\
\text { Mental calculation } \\
\text { Mental speech } \\
\text { Visual imagery }\end{array}$ \\
\hline Senden et al. (2019) & Visual imagery (four different letters) \\
\hline Yoo et al. (2007) & Motor imagery \\
\hline Lee et al. (2008) & Mental speech \\
\hline LaConte (2011) & $\begin{array}{l}\text { Emotion imagery (happy vs sad) } \\
\text { Mental speech (English vs Mandarin) } \\
\text { Motor imagery (left hand vs right } \\
\text { hand) }\end{array}$ \\
\hline $\begin{array}{l}\text { Andersson et al. (2010, } \\
\text { 2011) }\end{array}$ & Visuospatial attention \\
\hline Sorger et al. (2014) & Somatosensory attention \\
\hline $\begin{array}{l}\text { Naci et al. (2013) and } \\
\text { Naci and Owen } \\
(2013)\end{array}$ & Auditory attention \\
\hline
\end{tabular}

\section{MODUlation OF TEMPORAL fMRI-SIGNAL FEATURES}

As a second approach to distinctly encode separate intentions of BCI users, researchers have investigated the possibility of systematically varying temporal BOLD-signal features, i.e., allocating specific encoding time intervals (within a general encoding period) to specific intentions (Sorger, 2010; Bardin et al., 2011; Sorger et al., 2012). This approach was based on the observation of the authors that the onsets, offsets, and (thereby) durations of single-trial fMRI responses evoked by various mental activities can be reliably detected even when looking at trial time course plots from regions/networks of interest.

\section{Modulation OF Magnitudinal BOLD-Signal FEATURES}

As a third option, our research group explored the feasibility to encode separate intentions by reaching different fMRI-signal levels within a particular brain region. In an early real-time fMRI-neurofeedback hyperscanning study, magnitudinal BOLD-signal features were employed in such a way that two interacting participants could play "brain pong" controlling the vertical position of their rackets by modulating the level of regional brain activation (Goebel et al., 2004). Recently, this idea was investigated more systematically to answer the question how many different brain-activation levels could be reached with and without providing neurofeedback information about the current brain-activation level (Sorger, 2010; Krause et al., 2016; Zilverstand et al., 2017; Sorger et al., 2018). Interestingly, the ability to differentially modulate the BOLD-signal level was present even without providing neurofeedback when instructing participants carefully (i.e., providing appropriate modulation strategies). However, providing neurofeedback on the current brainactivation level further enhanced the gradual selfregulation performance (Sorger et al., 2018). Up to five different brain-activation levels (Sorger, 2010) could be differentiated. Note however, that this result was only obtained when averaging across a considerable number of trials. Trial averaging, of course, results in a considerably lower information transfer rate. However, the suggested approach might still constitute a valuable option for patients who do not have any other communication and control means left.

\section{Combinatory MODUlation OF BOLD-Signal FEATURES}

A general goal of $\mathrm{BCI}$ development is to maximize the degrees of freedom for the BCI user to encode different intentions, i.e., to allow the BCI user to choose from as many as possible options. One necessity in this context is to enable the BCI user to voluntarily evoke just as many 
differentiable mental states (and thereby distinct fMRIactivation patterns). However, as discussed earlier, it is very likely that only a few variations within the aforementioned BOLD signal-feature categories (spatial, temporal, and magnitudinal) are reliably differentiable in single (or few) trials. However, their combinatory use could constitute a possibility to either further increase the degrees of freedom in encoding different information units or to increase the differentiability of the evoked brain-activation patterns and thereby maximize decoding accuracies. The combinatory use of spatial and temporal BOLD-signal features was successfully tested in a multiple-choice paradigm obtaining a mean answerdecoding accuracy of $94.9 \%$ (theoretical chance level: $25.0 \%$ ) (Sorger et al., 2009). This approach was further developed to allow for en- and decoding all letters of the English alphabet and the blank space enabling fMRIbased free-letter spelling achieving a mean letter-decoding accuracy of $82 \%$ (theoretical chance level: ca. 3.7\%) (Sorger et al., 2012). Note however, that for encoding one individual multiple-choice answer or letter approximately 1 min was necessary.

\section{Information decoding using real-time fMRI data analysis}

As described above, a BCI based on volitional modulation of brain signals requires a specific translation code shared by the BCI user for encoding and the BCI system for decoding. Open source (Hinds et al., 2011; Koush et al., 2017) and commercial software (Turbo-BrainVoyager, Brain Innovation BV, Maastricht, The Netherlands) to decode the transmitted intention or message of the BCI user have been developed in the past analyzing regional or whole-brain activity in real-time.

\section{REAL-TIME fMRI DATA ANALYSIS}

To enable BCI applications, the measured fMRI data needs to be processed online during functional scanning. Data analysis should preferentially operate in real-time, i.e., the analysis for a newly measured time point (providing one functional volume composed of all measured slices) should be completed before the data of the next time point becomes available. Real-time processing (as opposed to near real-time fMRI), thus, restricts processing time to a maximum duration that is defined by the temporal interval between successive functional volumes, which typically assumes values between $1-3 \mathrm{~s}$ (volume time-to-repeat [TR]). While preprocessing and whole-brain statistical analyses typically process the data from moment to moment, specific BCI decoding calculations often operate on data in a small temporal window aggregating several data points for parameter estimation. The requirement for incremental analysis in limited time windows contrasts with conventional fMRI analyses processing data offline, i.e., data analysis only starts after the fMRI scanning session has ended without specific restrictions in calculation time.

Data acquisition techniques and analysis software have improved considerably since the introduction of real-time fMRI (Cox et al., 1995). The first real-time fMRI setups provided limited processing capabilities, e.g., lacking motion correction or moment-to-moment statistical analysis. Recent real-time fMRI BCIs employ analysis pipelines that include almost all preprocessing (see Section "Using fMRI to measure brain activation") and analysis steps used in conventional offline analysis as well as specialized routines for region-of-interest (ROI) time course extraction, multivoxel pattern analyses (MVPA), and visualization routines (Caria et al., 2012; Weiskopf, 2012). To guarantee constant (and fast) processing time, univariate statistical data analysis is usually performed recursively, i.e., estimated statistical parameters are updated by the information arriving with the next available functional volume instead of estimating them using the whole available time course from the first volume up to the volume of the current time point (Bagarinao et al., 2006). If the whole time course is used, calculation time of conventional algorithms (e.g., correlation analysis) increases with growing data sets bearing the risk of lagging behind the incoming data at some point. Instead, incremental algorithms provide constant calculation time per data point (volume) and enable real-time processing even for very long functional scans.

While correlation analysis was used in early real-time fMRI studies (Cox et al., 1995), full (incremental) GLM analyses are now performed (Goebel, 2001; Smyser et al., 2001; Bagarinao et al., 2003; Weiskopf et al., 2004; Hinds et al., 2011). While the design matrix for main experimental conditions may be built in advance for a planned experiment, sophisticated implementations support building the design matrix incrementally allowing to incorporate real-time imaging and behavioral data as it becomes available. This allows, e.g., that trials are assigned "on the fly" to specific experimental conditions with respect to trial-by-trial performance of the participant in the scanner. Incrementally built design matrices also allow incorporation of parameters obtained volume by volume from a 3D motion correction routine that may help to reduce residual motion artifacts. The real-time GLM design matrix may also contain confound predictors to model drifts in voxel time courses. Basic lowfrequency drift removal can be achieved by adding a linear trend predictor; for nonlinear trends, discrete cosine transform (DCT) confound predictors may be incrementally added to the design matrix. Removing drifts is especially important for BCI studies to ensure 
that increases or decreases of activity are caused by mental tasks and are not the result of unrelated signal drifts.

Note that the described (incremental) statistical analyses are usually operating at the single voxel level providing dynamic whole-brain statistical maps that integrate information of the whole voxel time course data from the start of the functional scan up to the currently processed volume. It is also possible to restrict the calculation of statistical values to a sliding window; depending on the specified size of the sliding window, obtained results reflect more dynamic changes (short sliding window) or more stable effects (large sliding window). While the calculation of whole-brain voxel-wise maps are not strictly necessary when one is interested in region-specific BCI effects (see below), whole-brain maps are very useful during functional scanning serving as a quality assurance tool allowing, e.g., to inspect activity in emotion, attention, and control networks indicating whether the participant is engaging in mental tasks.

\section{DeCODING BASED ON MULTIREGIONAL STATISTICAL ANALYSES}

Depending on the encoding/decoding approach (e.g., mental task A for "yes," mental task B for "no") contributing signals are restricted to relevant regions/networks and time windows. Regions are usually determined in a separate localizer run where participants engage in the relevant mental tasks prior to the actual BCI runs. When contrasting a specific task to baseline or to other tasks, the voxels with supra-threshold activity form a task-specific ROI or functional network. While the specification of task-related ROIs can be automatized (Lührs et al., 2017), this step is often performed by an experienced experimenter. Relevant regions/networks underlying specific mental tasks can also be determined using a priori (atlas) knowledge but the use of functional localizer runs usually provides higher signal modulation values and lower noise fluctuations than when using anatomical ROIs since region selection is optimized to the brain and specific task of the participant. The time course data for decoding events are usually extracted from restricted temporal windows that start with a rest period to calculate an up-to-date baseline signal level followed by a subsequent active mental task period. The windowed analysis approach with a rest and mental task period of 10-30s has the advantage that slow drifts have minimal impact on the BCI signal since all relevant time points are in close temporal proximity.

When decoding, to find which of multiple regions is most active, windowed time courses are used to calculate modulation estimates for each region separately using a single-trial GLM at the end of a BCI (i.e., mental-task) event. For the GLM, a specific trial-based design matrix is created containing a predictor modeling the expected hemodynamic response shape when the participant is engaging in a mental task, i.e., the time course shape is an idealized version of the one shown in Fig. 21.1 adjusted to the duration of the pre- and postbaseline and the duration of task performance. Additional confound time courses can be added such as a linear drift predictor. The estimated $\beta$ (or $t$ ) values of the task modulation predictor from each ROI are finally compared and the ROI with the largest modulation value is selected. The selected ROI thus provides a guess of the performed mental task and the associated intention of the BCI user can be retrieved based on the shared translation code. The described regional statistical decoding approach can be adjusted easily to incorporate temporal and magnitudinal features as described in Section "Information encoding in fMRI-based BCIs" increasing the number of intents that can be decoded from a single BCI trial.

\section{Decoding based on Multivoxel PatTern CLASSIFICATION}

While used extensively for EEG BCIs, multivariate machine learning techniques are gaining increasing interest for real-time fMRI BCI applications (Laconte et al., 2007; LaConte, 2011). One reason for the popularity of MVPA is based on its potential to detect differences between neural correlates of mental states with higher sensitivity as conventional statistical analyses since distributed activity patterns may reflect activity modulations better than ROI-based statistical approaches (see Section "Decoding based on multiregional statistical analyses"). Volunteers achieved, e.g., successful control of emotion-related activation patterns using a real-time support vector machine (SVM) classifier (Sitaram et al., 2011). While multivoxel pattern classifiers can be used in restricted regions, the approach may be especially useful to identify complex and interacting activity patterns over the whole brain (Laconte et al., 2007).

In such a multivariate approach, data from many sources (e.g., voxels in fMRI, channels in fNIRS, and EEG) are jointly analyzed to decode (predict) specific mental states or representational content. After performing a training phase, the decoding/prediction phase requires little computational load and it is, thus, suitable for real-time BCI applications including the decoding of mental states. In real-time fMRI MVPA is typically based on the widely used SVM learning algorithm producing a very good generalization performance. The SVM classifier is trained on data from one or more completed runs of a real-time session learning to associate different brain patterns with corresponding mental tasks according to the employed encoding/decoding translation code. The learning phase allows flexible adjustment to a specific 
brain by estimating weight values that differentiate patterns underlying two or more mental tasks performed by a participant. As opposed to EEG BCIs, it is important to note that only a few repetitions of mental task performance are usually necessary to successfully train a SVM classifier. After the training phase, online classification is turned on for $\mathrm{BCI}$ runs producing prediction values based on the estimated classifier weights that indicate to which class a generated distributed activity pattern belongs.

To obtain input patterns for different classes during training and testing, response values are estimated for each individual trial at each voxel (feature). Estimated single-trial responses across voxels then form the feature vectors used to train or test the classifier. An estimated trial response might be as simple as the activity level at a certain time point (e.g., at the time of the expected hemodynamic peak response) or the mean response of a few measurement points around the peak response relative to a prestimulus baseline. More robust estimates are obtained by integrating time points using single-trial GLM estimation (see earlier text). The estimated singletrial responses ( $\beta$ or $t$ values) across voxels form multivariate patterns that can be used for training runs and for online classification at the end of a trial. An attractive property of MVPA is that the classifier is adaptive and thus able to adjust to the participants' (changing) brain patterns during long-term BCI usage.

\section{OTHER MULTIVARIATE DECODING APPROACHES: ICA AND CONNECTIVITY}

Besides univariate statistics and distributed pattern analyses, data-driven multivariate analysis tools may provide important complementary real-time information. As an example, windowed independent component analysis (ICA) has been introduced for real-time fMRI analysis (Esposito et al., 2003) allowing to detect and visualize dynamic activity changes in functional brain networks that occur at unpredictable moments during a real-time fMRI experiment. Real-time windowed ICA might thus allow BCIs that do not require strict temporal guidance (e.g., "on" vs "baseline" epochs).

Another possibility is to use functional or effective connectivity measures as signatures for BCI events instead of voxel or regional mean activation levels. Functional connectivity refers to undirected coupling strength between voxels or regions and is usually calculated using standard correlation measures. Effective connectivity attempts to estimate directed modulatory effects between notes of a designed model (Koush et al., 2017). Different information content could be assigned to stronger or weaker coupling between brain regions instead of (only) the mean activation level of the involved regions. The online estimation of functional or effective connectivity between two or more brain regions requires, however, that a sufficiently long sliding window is used to calculate robust "instantaneous" coupling strength values. In a recent offline study, it has been shown that time windows of about 20 time points are sufficient to calculate robust (partial) correlation coefficients (Zilverstand et al., 2014). This study showed (in the context of various uni- and bimanual motor tasks) that instantaneous functional correlations may indeed provide relevant and unique information, which is not captured equally well by standard activation-based measures, regarding ongoing brain processes. Since estimation of functional and especially effective connectivity measures requires larger temporal windows than activity and MVPA approaches, such BCIs will benefit from recent technical advances such as accelerated simultaneous multislice imaging sequences (see Sections "Using fMRI to measure brain activation" and "Conclusions and future methodological perspectives of real-time fMRI”).

\section{CURRENT AND FUTURE REAL-TIME FMRI-BASED BCI APPLICATIONS}

The possibility of real-time fMRI-based brain-computer interfacing enriches the current spectrum of BCI systems. Its direct application in the context of brain-based communication and control for paralyzed patients is limited to only a few exceptional situations due to the drawbacks mentioned above (see Section "Evaluation of fMRI as BCI method"), especially the nonportability and challenging methodology. However, its noninvasive, relatively fast-to-apply nature and the general availability of MRI scanners in clinical environments make it certainly a to-be-considered candidate for applying fMRI-based communication, e.g., in acute stages of the "locked-in" syndrome (LIS) when other communication means are not available yet or in patients who cannot control other (e.g., neuroelectric) BCI systems (Sorger et al., 2012). Moreover, fMRI-based BCIs can detect online "neural behavior" and therewith serve as a crucial diagnostic tool to assess preserved conscious awareness in nonresponsive patients or to monitor the progress of disease in patients suffering from a disorder of consciousness (Owen et al., 2006; Monti et al., 2010). Of course, the clinical use of the fMRI-based BCI approach is not suited for prolonged time periods. Finally, we would like to mention the enabling function of fMRIbased BCIs for the development of practically more applicable communication and control BCIs using portable fNIRS, a method that is also based on the hemodynamic brain response.

Another promising field of application for fMRIbased brain-computer interfacing is its use as a neurofeedback technique. For example, real-time fMRI 
neurofeedback therapy is an emerging noninvasive neuromodulatory approach that is currently being investigated for its clinical potential: By providing information about ongoing locally specific brain activation related to brain disorder and dysfunction, patients get enabled to "self-regulate" pathologic brain processes into a desired direction and, thereby, to alleviate neurologic and psychiatric symptoms. Numerous translational studies explored the feasibility and effectiveness of real-time fMRI-neurofeedback to remediate pathologic brain activation associated with symptoms of neurologic and psychiatric disorders including major depressive disorder (Linden et al., 2012; Hamilton et al., 2016; Young et al., 2017), attention deficit hyperactivity disorder (Zilverstand et al., 2017), schizophrenia (Ruiz et al., 2008, 2013; Cordes et al., 2015; Dyck et al., 2016), Parkinson's disease (Subramanian et al., 2011; Linden and Turner, 2016), spider phobia (Zilverstand et al., 2015), chronic pain (deCharms et al., 2005; Chapin et al., 2012; Guan et al., 2015; Emmert et al., 2016), tinnitus (Haller et al., 2010; Emmert et al., 2017), addiction (Canterberry et al., 2013; Hanlon et al., 2013; Hartwell et al., 2013; Li et al., 2013; Karch et al., 2015; Kirsch et al., 2016), obesity (Frank et al., 2012), autism (Caria and de Falco, 2015), and stroke (Liew et al., 2016). Moreover, some studies explored the usefulness of fMRI-based neurofeedback training to enhance brain functions in healthy people (e.g., Shibata et al., 2011; Scharnowski et al., 2012). On the same lines, this methodology could be helpful to counteract age-related cognitive and motor decline (Rana et al., 2016). Most of these fMRI-based neurofeedback studies showed promising and encouraging results but further extensive research with appropriate control groups (Sorger et al., 2019) and careful evaluation ("follow-up" studies, costbenefit analyses etc.) is necessary.

Finally, we would like to stress the potential of realtime fMRI brain-computer interfacing as a promising neuroscientific research tool. One opportunity is the realization of experiments implementing brain statedependent stimulation. In such experiments, the content and/or the timing of the sensory stimulation is determined on the basis of ongoing brain activity. This methodology offers the possibility of addressing completely new research topics, including the investigation of causal brain-behavior relationships. Another auspicious neuroscientific application for fMRI-based brain-computer interfacing could be its use in hyperscanning studies. The decoding of brain states in real-time and the subsequent use of the resulting information in an ongoing experiment could provide an interesting methodology for the investigation of interacting brains in social situations.

\section{CONCLUSIONS AND FUTURE METHODOLOGICAL PERSPECTIVES OF REAL-TIME FMRI}

BCIs based on fMRI provide an important complement to other noninvasive BCIs. Despite its disadvantages (nonportable, methodologically challenging, costly, noisy environment), fMRI is the only method providing high-resolution whole-brain coverage of brain activation. These properties allow to relate mental tasks to specific regions and networks providing a transparent encoding/decoding scheme. The possibility of reaching deep into the brain allows to also use emotion processing areas such as the amygdala and ventral striatum, which are especially relevant for BCI neurofeedback applications in patients (Mehler et al., 2018). While fMRI BCIs' ultimate spatial and temporal resolution is limited by the physiologic properties of the hemodynamic response, technical and analytical advances may enable substantially improved fMRI BCIs in the future.

\section{High temporal resolution fMRI BCIs}

FMRI BCIs will benefit from recently introduced accelerated imaging methods such as simultaneous multislice (SMS) sequences (also known as "multiband" sequences) allowing a substantial increase in the number of collected data points per time unit (Feinberg and Yacoub, 2012). With appropriate multichannel head coils, these sequences allow the use of sampling times (volume TR) below $1 \mathrm{~s}$ while maintaining at or near whole brain coverage. More advanced techniques, such as magnetic resonance encephalography (MREG) may provide even sampling times of only $100 \mathrm{~ms}$ comparable to sampling times used in fNIRS BCI applications (Hennig et al., 2007; Assländer et al., 2013; Lührs et al., 2019). Unfortunately, the reconstruction of images from raw MREG data is, however, very computation intensive preventing its application to whole-brain real-time fMRI. A real-time compatible version limited to a priori selected regions of MREG is currently in development.

fMRI BCIs will substantially benefit from high temporal sampling in the order of $100-500 \mathrm{~ms}$ since major physiologic artifacts (related to respiration effects and cardiac pulsatility) can be removed by band-pass filtering, which is not possible with lower temporal resolution (aliasing). The explicit removal of physiologic nuisance effects will lead to much cleaner signals for BCI applications. High-temporal sampling also allows fMRI BCIs with shorter response delays because denser sampling of the hemodynamic response enables earlier detection of the onset of the BOLD-signal increase. Furthermore, analysis methods requiring many time points for robust 
estimates, such as partial correlation coefficients for functional connectivity, can be calculated 3-10 times faster than when using nonaccelerated sequences with volume TR (sampling) times of 1-2 s (see Section "Other multivariate decoding approaches: ICA and connectivity").

\section{High-spatial resolution fMRI BCIs at ultra-high magnetic fields}

In recent years, ultra-high field fMRI at $7 \mathrm{~T}$ or higher have provided novel "mesoscopic" neuroscience applications separating differential responses in cortical layers and columnar-like features inside small brain areas. The possibility to measure more detailed information may allow novel content-specific fMRI BCIs moving from patterns across task-related regions and networks to more fine-grained overlapping activation patterns within brain areas. Using multivoxel pattern classification at $7 \mathrm{~T}$, we have recently demonstrated that the direction of imagined motion (out of four options) can be identified with accuracies of up to $91.3 \%$ in individual subjects from activity in the early visual cortex (Emmerling et al., 2016). This result encourages the creation of fMRI BCIs based on subcategorical content.

The high signal-to-noise ratio at $7 \mathrm{~T}$ and higher may also be used to reliably pick up signals that are too weak for BCIs at conventional (1.5 and $3 \mathrm{~T})$ field strengths, such as top-down generated information during imagery of objects. We recently discovered (Senden et al., 2019) that it is indeed possible to reconstruct letter shapes from activity patterns in retinotopically organized early visual areas while participants merely imagined letter shapes during 7-T fMRI scanning (see Fig. 21.4). Reconstruction of a stimulus from brain activity patterns required only a short preparatory scan $(10 \mathrm{~min})$ to estimate the population receptive fields ( $\mathrm{pRFs}$ ) of activated voxels in the early visual cortex that relate points in visual space to locations in the early visual cortex (Dumoulin and Wandell, 2008). The reconstruction (decoding) process then inverts the established relationship projecting the pRFs of active voxels (location, size) back into the visual field. While the reconstructed letter images during imagery periods (upper row in Fig. 21.4) are not as clear as those decoded during perception of presented letters (middle and lower rows in Fig. 21.4), the correct letter could be reliably identified by spatially correlating the decoded image of an imagined letter with the images decoded from perceived letters. Importantly, we could demonstrate that imagined letter shapes can be decoded from single imagery events with a duration of only $6 \mathrm{~s}$ without the need to average across multiple repetitions. These observations encourage using letter imagery at $7 \mathrm{~T}$ as the basis for a more "natural" communication

Decoded images during letter imagery
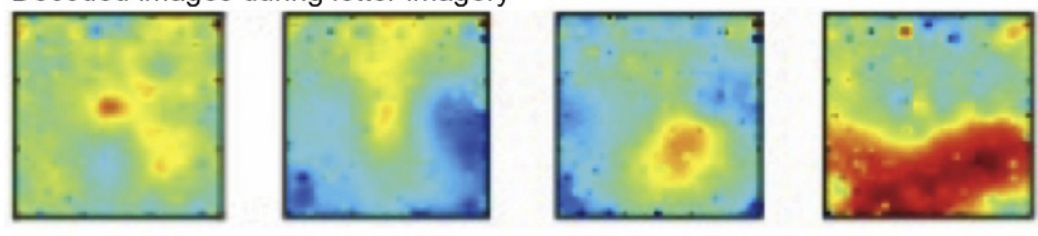

Decoded images during letter perception
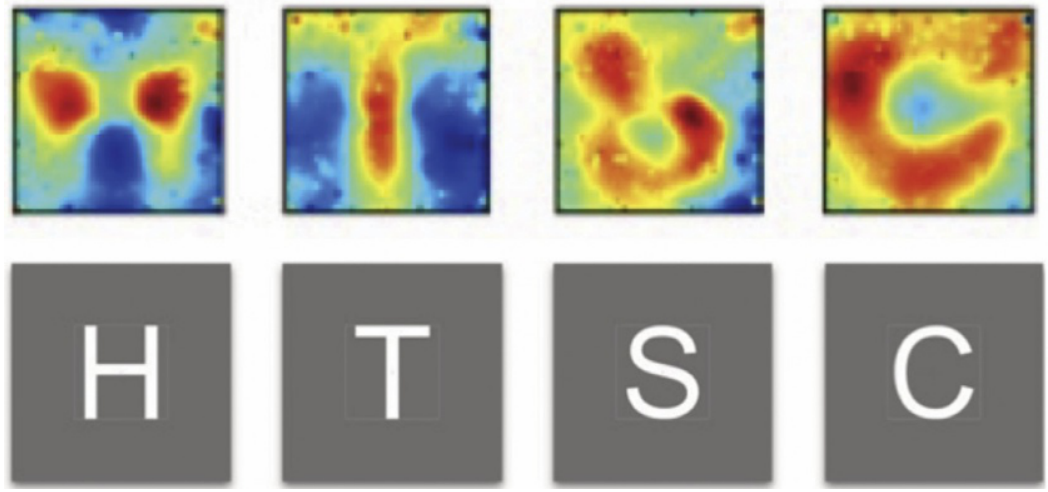

Fig. 21.4. Reconstructing seen and imagined letter shapes. Decoding seen and imagined letter shapes from brain activity patterns in the early visual cortex using 7-T fMRI (data from one participant). The visualization of seen and imagined letters is based on standard estimation of population receptive fields (pRFs) to relate positions in visual space to voxels in the early visual cortex (V1, V2, V3). The letters "H", "T", "S" and "C" were presented visually (bottom row) and the images in the middle row were reconstructed by projecting pRFs into visual space weighted by the activity of voxels. The upper row shows reconstructed letter shapes from top-down generated activity in the early visual cortex when the same participant merely imagined seeing the letter shapes. In this condition, letters were cued auditorily. 
fMRI BCI. Such a novel BCI could further benefit from presenting the online decoded letter to participants helping them to fine-tune the imagined letter shape interactively. In such a neurofeedback $B C I$ a first decoded image could be visually presented half way through an imagery period that is updated in the second half of the imagery phase by enhancing regions that receive increased top-down activation. This would allow participants to highlight critical regions of a letter during imagery without losing the generated overall letter shape.

\section{ACKNOWLEDGMENTS}

The authors gratefully acknowledge the support of the BrainGain Smart Mix Programme of the Netherlands Ministry of Economic Affairs and the Netherlands Ministry of Education, Culture and Science. This work was also supported by the Netherlands Organization for Scientific Research (NWO; RUBICON 446-09-010 awarded to B.S.) and the European Research Council (ERC; advanced grant \#269853 awarded to R.G.).

\section{REFERENCES}

Andersson P, Ramsey NF, Pluim JP et al. (2010). BCI control using 4 direction spatial visual attention and real-time fMRI at 7T. Conf Proc IEEE Eng Med Biol Soc 2010: 4221-4225.

Andersson P, Pluim JP, Siero JC et al. (2011). Real-time decoding of brain responses to visuospatial attention using 7T fMRI. PLoS One 6: e27638.

Assländer J, Zahneisen B, Hugger T et al. (2013). Single shot whole brain imaging using spherical stack of spirals trajectories. Neuroimage 73: 59-70.

Bagarinao E, Matsuo K, Nakai T et al. (2003). Estimation of general linear model coefficients for real-time application. Neuroimage 19: 422-429.

Bagarinao E, Nakai T, Tanaka Y (2006). Real-time functional MRI: development and emerging applications. Magn Reson Med Sci 5: 157-165.

Bardin JC, Fins JJ, Katz DI et al. (2011). Dissociations between behavioural and functional magnetic resonance imaging-based evaluations of cognitive function after brain injury. Brain 134: 769-782.

Birbaumer N, Murguialday AR, Cohen L (2008). Braincomputer interface in paralysis. Curr Opin Neurol 21: 634-638.

Bleichner MG, Jansma JM, Salari E et al. (2015). Classification of mouth movements using $7 \mathrm{~T}$ fMRI. J Neural Eng 12: 066026.

Bleichner MG, Freudenburg ZV, Jansma JM et al. (2016). Give me a sign: decoding four complex hand gestures based on high-density ECoG. Brain Struct Funct 221: 203-216.

Boly M, Coleman MR, Davis MH et al. (2007). When thoughts become action: an fMRI paradigm to study volitional brain activity in non-communicative brain injured patients. Neuroimage 36: 979-992.
Canterberry M, Hanlon CA, Hartwell KJ et al. (2013). Sustained reduction of nicotine craving with real-time neurofeedback: exploring the role of severity of dependence. Nicotine Tob Res 15: 2120-2124.

Caria A, de Falco S (2015). Anterior insular cortex regulation in autism spectrum disorders. Front Behav Neurosci 9: 38.

Caria A, Sitaram R, Birbaumer N (2012). Real-time fMRI: a tool for local brain regulation. Neuroscientist 18: 487-501.

Chapin H, Bagarinao E, Mackey S (2012). Real-time fMRI applied to pain management. Neurosci Lett 520: 174-181.

Cordes JS, Mathiak KA, Dyck M et al. (2015). Cognitive and neural strategies during control of the anterior cingulate cortex by fMRI neurofeedback in patients with schizophrenia. Front Behav Neurosci 9: 169.

Cox RW, Jesmanowicz A, Hyde JS (1995). Real-time functional magnetic resonance imaging. Magn Reson Med 33: 230-236.

De Martino F, Moerel M, Ugurbil K et al. (2015). Frequency preference and attention effects across cortical depths in the human primary auditory cortex. Proc Natl Acad Sci USA 112: 16036-16041.

deCharms RC, Maeda F, Glover GH et al. (2005). Control over brain activation and pain learned by using real-time functional MRI. Proc Natl Acad Sci USA 102: 18626-18631.

Dumoulin SO, Wandell BA (2008). Population receptive field estimates in human visual cortex. Neuroimage 39: 647-660.

Dyck MS, Mathiak KA, Bergert S et al. (2016). Targeting treatment-resistant auditory verbal hallucinations in schizophrenia with fMRI-based neurofeedbackexploring different cases of schizophrenia. Front Psych 7: 37 .

Emmerling TC, Zimmermann J, Sorger B et al. (2016). Decoding the direction of imagined visual motion using 7T ultra-high field fMRI. Neuroimage 125: 61-73.

Emmert K, Breimhorst M, Bauermann T et al. (2016). Active pain coping is associated with the response in real-time fMRI neurofeedback during pain. Brain Imaging Behav 11: 712-721.

Emmert K, Kopel R, Koush Y et al. (2017). Continuous vs. intermittent neurofeedback to regulate auditory cortex activity of tinnitus patients using real-time fMRI-a pilot study. Neuroimage Clin 14: 97-104.

Esposito F, Seifritz E, Formisano E et al. (2003). Real-time independent component analysis of fMRI time-series. Neuroimage 20: 2209-2224.

Feinberg DA, Yacoub E (2012). The rapid development of high speed, resolution and precision in fMRI. Neuroimage 62: 720-725.

Frank S, Lee S, Preissl H et al. (2012). The obese brain athlete: self-regulation of the anterior insula in adiposity. PLoS One 7: e42570.

Goebel R (2001). Cortex-based real-time fMRI. Neuroimage 13: S129.

Goebel R, Sorger B, Kaiser J et al. (2004). BOLD brain pong: self regulation of local brain activity during synchronously scanned, interacting subjects. In: 34th annual meeting of the society for neuroscience, San Diego, USA. 
Guan M, Ma L, Li L et al. (2015). Self-regulation of brain activity in patients with postherpetic neuralgia: a doubleblind randomized study using real-time FMRI neurofeedback. PLoS One 10: e0123675.

Haller S, Birbaumer N, Veit R (2010). Real-time fMRI feedback training may improve chronic tinnitus. Eur Radiol 20: 696-703.

Hamilton JP, Glover GH, Bagarinao E et al. (2016). Effects of salience-network-node neurofeedback training on affective biases in major depressive disorder. Psychiatry Res 249: 91-96.

Hanlon CA, Hartwell KJ, Canterberry M et al. (2013). Reduction of cue-induced craving through realtime neurofeedback in nicotine users: the role of region of interest selection and multiple visits. Psychiatry Res 213: 79-81.

Hartwell KJ, Prisciandaro JJ, Borckardt J et al. (2013). Realtime fMRI in the treatment of nicotine dependence: a conceptual review and pilot studies. Psychol Addict Behav 27: 501-509.

Hennig J, Zhong K, Speck O (2007). MR-encephalography: fast multi-channel monitoring of brain physiology with magnetic resonance. Neuroimage 34: 212-219.

Hinds O, Ghosh S, Thompson TW et al. (2011). Computing moment-to-moment BOLD activation for real-time neurofeedback. Neuroimage 54: 361-368.

Kaas A, Goebel R, Rosenke M et al. (2014). A somatotopically specific tactile imagery paradigm for fMRI brain computer interface applications. In: 20th Annual meeting of the organization for human brain mapping, Hamburg, Germany.

Karch S, Keeser D, Hummer S et al. (2015). Modulation of craving related brain responses using real-time fMRI in patients with alcohol use disorder. PLoS One 10: e0133034.

Kirsch M, Gruber I, Ruf M et al. (2016). Real-time functional magnetic resonance imaging neurofeedback can reduce striatal cue-reactivity to alcohol stimuli. Addict Biol 21: 982-992.

Koush Y, Ashburner J, Prilepin E et al. (2017). OpenNFT: an open-source python/Matlab framework for real-time fMRI neurofeedback training based on activity, connectivity and multivariate pattern analysis. Neuroimage 156: 489-503.

Krause F, Benjamins C, Luhrs M et al. (2016). Real-time selfregulation across multiple visual neurofeedback presentations. In: 6th International brain-computer interface meeting, Asilomar, USA.

LaConte SM (2011). Decoding fMRI brain states in real-time. Neuroimage 56: 440-454.

Laconte SM, Peltier SJ, Hu XP (2007). Real-time fMRI using brain-state classification. Hum Brain Mapp 28: 1033-1044.

Lee J, O'Leary H, Lee S et al. (2006). Automated spatiotemporal classification of human minds for braincomputer-interface. In: 36th Annual meeting of the society for neuroscience, Atlanta, USA.

Lee JH, O'Leary HM, Park H et al. (2008). Atlas-based multichannel monitoring of functional MRI signals in real-time: automated approach. Hum Brain Mapp 29: 157-166.

Lee JH, Marzelli M, Jolesz FA et al. (2009a). Automated classification of fMRI data employing trial-based imagery tasks. Med Image Anal 13: 392-404.
Lee JH, Ryu J, Jolesz FA et al. (2009b). Brain-machine interface via real-time fMRI: preliminary study on thoughtcontrolled robotic arm. Neurosci Lett 450: 1-6.

Li X, Hartwell KJ, Borckardt J et al. (2013). Volitional reduction of anterior cingulate cortex activity produces decreased cue craving in smoking cessation: a preliminary real-time fMRI study. Addict Biol 18: 739-748.

Liew SL, Rana M, Cornelsen S et al. (2016). Improving motor corticothalamic communication after stroke using real-time fMRI connectivity-based neurofeedback. Neurorehabil Neural Repair 30: 671-675.

Linden DE, Turner DL (2016). Real-time functional magnetic resonance imaging neurofeedback in motor neurorehabilitation. Curr Opin Neurol 29: 412-418.

Linden DE, Habes I, Johnston SJ et al. (2012). Real-time selfregulation of emotion networks in patients with depression. PLoS One 7: e38115.

Logothetis NK, Pauls J, Augath M et al. (2001). Neurophysiological investigation of the basis of the fMRI signal. Nature 412: 150-157.

Lührs M, Sorger B, Goebel R et al. (2017). Automated selection of brain regions for real-time fMRI brain-computer interfaces. J Neural Eng 14: 016004.

Lührs M, Riemenschneider B, Eck J et al. (2019). The potential of MR-encephalography for BCI/neurofeedback applications with high temporal resolution. Neuroimage 194: 228-243.

Mehler DMA, Sokunbi MO, Habes I et al. (2018). Targeting the affective brain-a randomized controlled trial of realtime fMRI neurofeedback in patients with depression. Neuropsychopharmacology 43: 2578-2585.

Moeller S, Yacoub E, Olman CA et al. (2010). Multiband multislice GE-EPI at 7 tesla, with 16-fold acceleration using partial parallel imaging with application to high spatial and temporal whole-brain fMRI. Magn Reson Med 63: 1144-1153.

Monti MM, Vanhaudenhuyse A, Coleman MR et al. (2010). Willful modulation of brain activity in disorders of consciousness. N Engl J Med 362: 579-589.

Naci L, Owen AM (2013). Making every word count for nonresponsive patients. JAMA Neurol 70: 1235-1241.

Naci L, Cusack R, Jia VZ et al. (2013). The brain's silent messenger: using selective attention to decode human thought for brain-based communication. J Neurosci 33: 9385-9393.

Ogawa S, Lee TM, Kay AR et al. (1990). Brain magnetic resonance imaging with contrast dependent on blood oxygenation. Proc Natl Acad Sci USA 87: 9868-9872.

Owen AM, Coleman MR, Boly M et al. (2006). Detecting awareness in the vegetative state. Science 313: 1402.

Owen AM, Coleman MR, Boly M et al. (2007). Using functional magnetic resonance imaging to detect covert awareness in the vegetative state. Arch Neurol 64: 1098-1102.

Rana M, Varan AQ, Davoudi A et al. (2016). Real-time fMRI in neuroscience research and its use in studying the aging brain. Front Aging Neurosci 8: 239.

Rauschenberg J, Nagel AM, Ladd SC et al. (2014). Multicenter study of subjective acceptance during magnetic resonance imaging at 7 and 9.4 T. Invest Radiol 49: 249-259. 
Ruiz S, Sitaram R, Soekadar SR et al. (2008). Learned control of insular activity and functional connectivity changes using a fMRI brain computer interface in schizophrenia. 38th Annual meeting of the society for neuroscience, Washington, USA.

Ruiz S, Lee S, Soekadar SR et al. (2013). Acquired self-control of insula cortex modulates emotion recognition and brain network connectivity in schizophrenia. Hum Brain Mapp 34: 200-212.

Scharnowski F, Hutton C, Josephs O et al. (2012). Improving visual perception through neurofeedback. J Neurosci 32: $17830-17841$.

Seitz RJ (2010). How imaging will guide rehabilitation. Curr Opin Neurol 23: 79-86.

Senden M, Emmerling TC, van Hoof R et al. (2019). Reconstructing imagined letters from early visual cortex reveals tight topographic correspondence between visual mental imagery and perception. Brain Struct Funct 224: 1167-1183.

Shibata K, Watanabe T, Sasaki Y et al. (2011). Perceptual learning incepted by decoded fMRI neurofeedback without stimulus presentation. Science 334: 1413-1415.

Sitaram R, Lee S, Ruiz S et al. (2011). Real-time support vector classification and feedback of multiple emotional brain states. Neuroimage 56: 753-765.

Smyser C, Grabowski TJ, Frank RJ et al. (2001). Real-time multiple linear regression for fMRI supported by timeaware acquisition and processing. Magn Reson Med 45: 289-298.

Sorger B (2010). When the brain speaks for itself: Exploiting hemodynamic brain signals for motor-independent communication, $\mathrm{PhD}$ thesis, Maastricht University.

Sorger B, Dahmen B, Reithler J et al. (2009). Another kind of 'BOLD Response': answering multiple-choice questions via online decoded single-trial brain signals. Prog Brain Res 177: 275-292.

Sorger B, Reithler J, Dahmen B et al. (2012). A real-time fMRI-based spelling device immediately enabling robust motor-independent communication. Curr Biol 22: 1333-1338.

Sorger B, Goebel R, Rosenke M et al. (2014). A novel paradigm for fMRI-based brain-computer interfacing using selective somatosensory attention. In: 20th Annual meeting of the organization for human brain mapping, Hamburg, Germany.

Sorger B, Kamp T, Weiskopf N et al. (2018). When the brain takes 'BOLD' steps: Real-time fMRI neurofeedback can further enhance the ability to gradually self-regulate regional brain activation. Neuroscience 378 : 71-88.
Sorger B, Scharnowski F, Linden DEJ et al. (2019). Control freaks: towards optimal selection of control conditions for fMRI neurofeedback studies. Neuroimage 186: 256-265.

Subramanian L, Hindle JV, Johnston S et al. (2011). Real-time functional magnetic resonance imaging neurofeedback for treatment of Parkinson's disease. J Neurosci 31: $16309-16317$.

Uludag K (2010). To dip or not to dip: reconciling optical imaging and fMRI data. Proc Natl Acad Sci USA 107: E23. author reply E24.

Weiskopf N (2012). Real-time fMRI and its application to neurofeedback. Neuroimage 62: 682-692.

Weiskopf N, Mathiak K, Bock SW et al. (2004). Principles of a brain-computer interface (BCI) based on real-time functional magnetic resonance imaging (fMRI). IEEE Trans Biomed Eng 51: 966-970.

Weiskopf N, Hutton C, Josephs O et al. (2006). Optimal EPI parameters for reduction of susceptibility-induced BOLD sensitivity losses: a whole-brain analysis at $3 \mathrm{~T}$ and 1.5 T. Neuroimage 33: 493-504.

Yoo SS, Lee CU, Choi BG (2001). Human brain mapping of auditory imagery: event-related functional MRI study. Neuroreport 12: 3045-3049.

Yoo SS, Freeman DK, McCarthy 3rd JJ et al. (2003). Neural substrates of tactile imagery: a functional MRI study. Neuroreport 14: 581-585.

Yoo SS, Fairneny T, Chen NK et al. (2004). Brain-computer interface using fMRI: spatial navigation by thoughts. Neuroreport 15: 1591-1595.

Yoo SS, O'Leary HM, Lee JH et al. (2007). Reproducibility of trial-based functional MRI on motor imagery. Int J Neurosci 117: 215-227.

Young KD, Siegle GJ, Zotev V et al. (2017). Randomized clinical trial of real-time fMRI amygdala neurofeedback for major depressive disorder: effects on symptoms and autobiographical memory recall. Am J Psychiatry 174: 748-755. appiajp201716060637.

Zilverstand A, Sorger B, Zimmermann J et al. (2014). Windowed correlation: a suitable tool for providing dynamic fMRI-based functional connectivity neurofeedback on task difficulty. PLoS One 9: e85929.

Zilverstand A, Sorger B, Sarkheil P et al. (2015). fMRI neurofeedback facilitates anxiety regulation in females with spider phobia. Front Behav Neurosci 9: 148.

Zilverstand A, Sorger B, Slaats-Willemse D et al. (2017). fMRI neurofeedback training for increasing anterior cingulate cortex activation in adult attention deficit hyperactivity disorder. An exploratory randomized, single-blinded study. PLoS One 12: e0170795. 